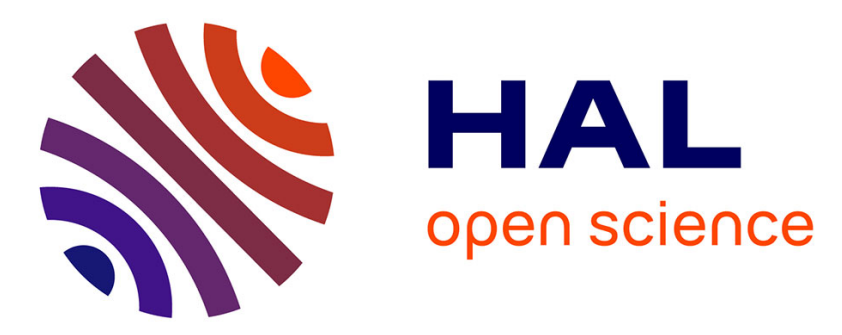

\title{
Circonscriptions de police et souveraineté territoriale: les premières semaines de Rome capitale dans les mémoires du commissaire Manfroni
}

Denis Bocquet

\section{- To cite this version:}

Denis Bocquet. Circonscriptions de police et souveraineté territoriale : les premières semaines de Rome capitale dans les mémoires du commissaire Manfroni. Mélanges de l'École française de Rome - Italie et Méditerranée, 2003, 2 (115), pp.845-862. halshs-00128708

\section{HAL Id: halshs-00128708 \\ https://shs.hal.science/halshs-00128708}

Submitted on 27 Mar 2007

HAL is a multi-disciplinary open access archive for the deposit and dissemination of scientific research documents, whether they are published or not. The documents may come from teaching and research institutions in France or abroad, or from public or private research centers.
L'archive ouverte pluridisciplinaire HAL, est destinée au dépôt et à la diffusion de documents scientifiques de niveau recherche, publiés ou non, émanant des établissements d'enseignement et de recherche français ou étrangers, des laboratoires publics ou privés. 
Circonscriptions de police et souveraineté territoriale : les premières semaines de Rome capitale dans les mémoires du commissaire Manfroni

\section{Denis Bocquet}

Texte publié dans les Mélanges de l'Ecole française de Rome (Italie et Méditerranée) (MEFRIM), 2003, vol. 2, nº 115, p. 845-862

Les premiers temps de la souveraineté de l'Etat italien dans la ville pontificale après les événements du 20 septembre 1870 sont marqués par une forte incertitude quant aux limites territoriales de cette souveraineté face au Vatican. Pendant plusieurs semaines, voire plusieurs mois, plane sur les divers services de l'Etat italien un flou persistant dans la définition de leur aire de compétence sur la ville à peine conquise. $\mathrm{Si}$, au moment de la bataille du 20 septembre, il est d'emblée évident pour le gouvernement italien qu'il convient de laisser au Pape et à ses troupes la possibilité d'un repli vers le Vatican et ce qu'on appelle la cité Léonine, et s'il est encore plus évident que cette place forte doit leur être concédée de manière pérenne, de nombreuses questions demeurent en suspend après la prise de la ville : quelle définition territoriale de la cité Léonine adopter? Quelle partie de Rome autour de celle-ci laisser à Pie IX ? Jusqu'où étendre l'application 
de l'ordre administratif italien qui progressivement se met en place? Comment gérer les susceptibilités catholiques sans aliéner les prétentions italiennes à la pleine souveraineté sur la ville?

Dans un contexte international extrêmement tendu, où chaque initiative du gouvernement italien soulève de véhémentes protestations de la part des grandes puissances catholiques, il est intéressant de suivre dans le détail la manière dont les fonctionnaires de l'Etat, soldats, policiers ou ingénieurs, prennent pied dans la ville destinée à devenir rapidement la nouvelle capitale du royaume, selon le vœu de Cavour et les décisions du Parlement au début des années 1860 .

La question territoriale à Rome a aussi pour origine le déroulement même des événements qui ont conduit à la prise de la ville. Les opérations militaires sont souvent décrites comme purement anecdotiques. Le 20 septembre, les armées italienne et pontificale se sont néanmoins livrées une réelle bataille ${ }^{1}$. L'été avait été marqué par les tractations franco-italiennes qui ont précédé le déclenchement de la guerre avec la Prusse. Napoléon III recherchait l'alliance avec l'Italie, mais refusait de se retirer de Civitavecchia. L'Italie aurait souhaité une alliance avec la France et avec l'Autriche-Hongrie, mais au prix de Rome et du Trentin. Lorsqu'en août Napoléon III s'était déclaré prêt à faire des concessions, il était déjà trop tard: la France avait déjà subi ses premières défaites à l'Est, et l'Italie n'avait plus aucun intérêt à mettre sa sécurité nationale en danger pour 
obtenir un avantage que le cours des événements lui donnerait sans contrepartie. A la fin du mois d'août, le gouvernement italien rassemble plusieurs dizaines de milliers d'hommes en Ombrie, au nord de la frontière avec l'Etat pontifical. G.B. Calogero souligne l'ambiguité de ce «corps d'observation de l'Italie centrale »: il pouvait tout autant s'agir d'une manœuvre destinée à empêcher toute action républicaine contre Rome, que de la base arrière d'une expédition dirigée contre la ville éternelle le long des voies consulaires Cassia, Salaria et Flaminia ${ }^{2}$. A la tête de ce corps, qui ne prend le nom de Quarto Corpo d'Esercito qu'une fois l'invasion décidée, le gouvernement place le général Cadorna, vétéran des guerres de Crimée et d'Indépendance nationale.

Au tournant du mois de septembre, la défaite de la France devient totale. Le gouvernement florentin envoie à Rome San Martino pour négocier une entrée sans combat des troupes italiennes dans l'Etat du Pape et dans Rome, mais Pie IX refuse de négocier. Le Pape, depuis le départ des Français, disposait d'une armée de 13000 hommes, ${ }^{3}$ dirigée par le général bavarois Kanzler, assisté de Kalbermatten, De Courten et Zappi. Rome était aussi défendue par ses murailles, mais sur $22 \mathrm{~km}$, seuls 7 , essentiellement sur la rive droite du Tibre, étaient renforcés par des bastions modernes. Pour le reste, les antiques murs d'Aurélien étaient nus. Kanzler place en septembre l'essentiel des troupes à Rome. Certaines portes avaient été murées (San Lorenzo, Porta Maggiore, San Sebastiano, San Paolo, San Pancrazio, 
Cavalleggeri). L'état d'urgence est déclaré dans la ville à partir du 12 septembre.

Les forces italiennes étaient nettement plus nombreuses dès le mois d'août. En tout, elles comptent en septembre plus de 52000 hommes, 7500 chevaux et 114 canons.

Du 11 au 15 septembre, le gouvernement italien procède à des manœuvres plus démonstratives qu'offensives, pour tenter de convaincre le Pape de renoncer à faire défendre la ville. Le 15, l'émissaire du général, Caccialupi, reçoit une réponse négative de Kanzler aux propositions de reddition ${ }^{4}$. Le 17, une ultime médiation du comte d'Arnim, représentant prussien auprès du Saint-Siège, échoue. C'est le 18 au soir que, par télégraphe, le gouvernement donne l'ordre, depuis Florence, d'attaquer Rome. Le 19 septembre, Pie IX Mastai ordonne au général Kanzler d'opposer une résistance brève, mais ferme, afin de montrer au monde que c'est par la force que la ville sera prise ${ }^{5}$. Au Parlement de Turin, la gauche, derrière Mancini, exhorte le gouvernement à passer à l' action ${ }^{6}$.

L'attaque de la ville est prévue sur la rive gauche, là où les murs sont les plus faibles, mais aussi de manière à être sûr de ne pas endommager le Vatican, tout en permettant aux troupes pontificales de s'y retirer. L'essentiel de l'attaque est concentré à hauteur de Porta Salaria et Porta Pia, sur le plateau des hauteurs de la ville, sur la rive gauche. Bixio, revenu de Civitavecchia, est chargé d'attaquer Trastevere dans un second temps, avec 
l'ordre strict de ne causer aucun dommage à la Città Leonina. On voit que dès cet instant se dessine la future partition de souveraineté sur la ville. Les plans militaires d'attaque pour le 20 septembre, conformément aux instructions du gouvernement, prévoient déjà non seulement une voie honorable et sûre de retraite pour les troupes pontificales, mais aussi esquissent les limites d'une zone refuge pour le Pape, sa cour et son armée. Dès 10 heures du matin, le 20 septembre, les troupes pontificales hissent le drapeau blanc, mais attendent le soir pour ouvrir les portes, afin de permettre la retraite vers le Vatican des combattants de la rive gauche. L'assaut de la Porta San Giovanni, au sud, par le général Angioletti donne le même résultat.

L'essentiel des combats a lieu à Porta Pia, où est ouverte une brèche de 30 mètres dans les murs antiques, grâce aux tirs de l'artillerie. 25000 Italiens, essentiellement bersaglieri, font face à 800 pontificaux. Au bout de plusieurs heures d'intenses combats, les zouaves pontificaux se rendent ou se retirent vers Saint-Pierre. Le bilan de la journée est lourd cependant pour une opération dont l'histoire n'a retenu que l'aspect anecdotique: 48 soldats italiens, et 16 pontificaux sont morts. On compte au total près de 200 blessés.

La prise de Rome par les troupes italiennes connaît un grand écho sur la scène internationale. Les protestations sont nombreuses, et affluent de tout le monde chrétien, France, Belgique, Espagne et Canada au premier chef ${ }^{7}$. 
D'un point de vue diplomatique cependant, comme le montre C.M. Fiorentino, les capitales de pays catholiques avaient été beaucoup plus modérées face aux avancées italiennes vers Rome. Durant tout le mois de septembre 1870, le Ministre Visconti-Venosta, pour le gouvernement italien, s'était efforcé de rassurer. Il avait, semble-t-il, été entendu, y compris à Munich, Bruxelles, Paris et Vienne. A Paris, c'est Jules Favre, ministre des Affaires étrangères du gouvernement de Défense nationale, qui avait fait part à l'ambassadeur italien de son indifférence face au sort du pouvoir temporel pontifical. La Prusse, quant à elle, pousse ouvertement l'Italie à prendre Rome. Pour le gouvernement de Florence, la voie était donc libre sur le plan diplomatique.

A partir du 20 septembre cependant, la situation se tend de nouveau, avec les protestations véhémentes de Pie IX et la mobilisation d'une frange importante des opinions catholiques. Le Pape crie en effet au sacrilège, de même que le cardinal Antonelli, qui fait parvenir une circulaire aux représentants diplomatiques étrangers auprès du Saint-Siège ${ }^{8}$. La presse catholique se fait également l'écho de l'indignation du souverain pontife. Pendant de nombreuses années, des pèlerins catholiques, français essentiellement, mènent aux abords du Vatican des manifestations bruyantes de dénonciation de la spoliation, voire de provocation face aux forces de l'ordre italiennes ${ }^{9}$. 
Un certain nombre d'incidents marquent les premiers temps de la présence italienne en ville. La presse catholique du monde entier dénonce largement la liberté laissé par La Marmora à des exilés romains révolutionnaires de retour dans la ville avec des idées de vengeance face à la Papauté. Le 20 Octobre, Pie IX fait afficher un bref sur les basiliques de la ville, informant de la suspension des travaux du Concile : « sacrilega repente invasio huius Almae Urbis, Sedis Nostrae, et reliquarum temporalis Nostrae ditionis regiorum, qua contra omne fas civilis Nostri et Apostolicae Sedis Principatus inconcussa iura incredibili perfidia et audacia violata sunt $»^{10}$. L'aspect le plus significatif de la protestation pontificale reste cependant le refus du Pape, transmis à tous les échelons de la hiérarchie catholique, de traiter avec un pouvoir considéré comme usurpateur. Durant les premiers mois qui suivent le 20 septembre, les bureaux de la Papauté emploient pour désigner le gouvernement italien le terme Subalpinium Gubernium, qui renvoie au Piémont, et non au Royaume d'Italie. Même si, jusqu'au 7 octobre, les contacts informels entre le cardinal Antonelli et les émissaires du gouvernement sont maintenus, rapidement c'est la ligne dure voulue par Pie IX lui-même qui triomphe.

La préparation de la loi sur les Garanties se fait donc dans un climat de grande tension. En mai 1871, par l'encyclique Ubi Nos, Pie IX repousse le texte que le Parlement italien vient de voter afin d'assurer la Papauté de l'indépendance du pouvoir spirituel et de moyens de subsistance. 
Pour l'Eglise catholique, la fin du pouvoir temporel du Pape constitue un moment important de redéfinition de nombreuses grandes orientations. Aux protestations, font place peu à peu d'intenses réflexions sur l'avenir de l'Eglise ${ }^{11}$. Ce qui marque fortement les premiers mois et les premières années est cependant un choc face à la disparition de l'assise territoriale et étatique pluriséculaire de la Papaute ${ }^{12}$. Comme le montre Giacomo Martina, dans la conscience catholique, demeure longtemps présente l'idée d'un retour en arrière face à la perte de Rome. Aux premières réactions dénonçant le sacrilège, succèdent des actions plus marquées dans le sens de l'organisation d'une protestation ${ }^{13}$. Au moment du plébiscite pour le rattachement de Rome au royaume d'Italie, une pétition de 4000 dames de l'aristocratie et de la bourgeoisie est réunie, ainsi qu'une pétition, à l'initiative de la Società romana per gli interessi cattolici, rassemblant les signatures de plus de 27000 citoyens $^{14}$. Mais dès la fin de l'année 1870 et le début de l'année 1871, les Catholiques intransigeants choisissent une autre ligne de conduite face aux autorités italiennes : l'auto-exclusion de la vie politique nationale. Pour de nombreuses années, ils cessent d'avoir des rapports officiels avec les instances gouvernementales italiennes, et refusent de participer aux élections nationales ${ }^{15}$.

Du côté italien, le rattachement ouvre de nombreuses perspectives inédites et l'entrée des troupes italiennes dans la ville pose de nouveaux problèmes $^{16}$. Le premier est d'ordre pratique et politique, autour de la 
décision de transférer la capitale. Le second est d'ordre territorial: où s'arrête la Rome italienne, et quelle définition de la Città Leonina dévolue au Pape sera retenue?

Les clauses de la capitulation signée par le général Kanzler le 20 septembre stipulent que l'Italie laisse au Pape la Città Leonina. Mais rien n'indique quelle interprétation donner à cette expression. Il semblerait que le 21 septembre le cardinal Antonelli ait même demandé à Cadorna d'occuper le Vatican, après des incidents au abords de Saint-Pierre ${ }^{17}$. La volonté du cardinal était sans doute d'amener l'Italie à aller au-delà de la ligne qu'elle s'était fixée comme raisonnable, et à mettre un peu plus le gouvernement dans l'embarras. Cadorna ne tombe pas dans le piège. La question demeure cependant de savoir où s'arrête la nouvelle Italie.

La question de l'étendue territoriale de la zone laissée au Pape occupe les premières semaines, voire les premiers mois de la présence des autorités italiennes à Rome. La lecture des mémoires du commissaire Manfroni en donne l'idée la plus précise. Envoyé d'abord à Trastevere dans les jours qui suivent le 20 septembre, puis dans le quartier du Borgo, au contact du Vatican, le commissaire est l'homme de confiance du ministre de l'Intérieur pour la gestion quotidienne et pragmatique de la question territoriale. Dans ses mémoires, on peut suivre au jour le jour le cheminement des autorités italiennes sur la rive droite, et surtout les nombreuses hésitations et approximations à ce sujet ${ }^{18}$. 
Dans les premiers jours après le 20 septembre, les troupes italiennes hésitent longuement avant de traverser les ponts sur le Tibre et d'investir la rive droite du fleuve, celle du Vatican et de la Città Leonina. La prise de Trastevere est certes précoce, mais les Italiens tardent à y afficher les symboles de leur souveraineté. Mafroni montre combien, au cours des premières semaines, la police italienne est discrète, et renonce à parader dans le quartier, et même à patrouiller en uniforme. Ce n'est que peu à peu, l'automne avançant, que les fonctionnaires italiens parviennent à se faire une place face à des gendarmes pontificaux qui continuent de patrouiller en civil.

Pour ce qui concerne les abords du Vatican, la situation est encore plus complexe. Non seulement les fonctionnaires ne disposent d'aucune indication gouvernementale sur l'étendue de la Città Leonina à laisser au Pape, mais, pendant plusieurs semaines, de nombreuses hypothèses circulent, dont celle de laisser au Pape une bande de terre allant jusqu'à la mer, ou de lui laisser le quartier du Borgo. Le sort de la place Saint-Pierre et de la basilique reste flou pendant de longs mois: elles sont hors des murailles du Vatican, mais constituent en même temps l'espace le plus fort de la symbolique vaticane.

Manfroni adopte à ce sujet à l'automne 1870 la plus sage des attitude : il considère que la place et la basilique sont en Italie, mais prend soin d'y 
patrouiller lui-même et en civil, évitant d'y envoyer des policiers italiens en uniforme.

Dans ce contexte, le plébiscite du 2 octobre 1870 est pour le sort de la rive droite un moment délicat. Le Borgo n'est pas inclus en effet dans l'organisation du vote du 2 octobre 1870 devant sanctionner le rattachement de Rome et de l'Etat pontifical à l'Italie. Des habitants, patriotes zélés et désireux de malgré tout ratifier l'appartenance de leur quartier à l'Italie, organisent cependant la tenue d'un bureau de vote, et les résultats sont ensuite insérés dans le décompte global. Près de deux semaines après la prise de la ville, on est encore dans une période de tâtonnements.

Le 9 octobre 1870 un décret de Victor Emmanuel déclare l'annexion ${ }^{19}$ :

Art. 1: Rome et les provinces romaines sont parties intégrantes du Royaume d'Italie

Art. 2 : Le Souverain Pontife conserve la dignité, l'inviolabilité et toutes les prérogatives personnelles d'un souverain

Art. 3: Une loi viendra déterminer les garanties et les franchises territoriales destinées à assurer l'indépendance du Souverain Pontife, ainsi que le libre exercice de l'autorité spirituelle du Saint-Siège

La question territoriale demeure en suspens. Depuis l'acte de capitulation entre Kanzler et Cadorna, lieutenant général du Roi à Rome, qui pourtant était assez imprécis, rien n'a été clarifié. Le 20 septembre, à villa Albani 
(devenue ensuite Torlonia, sur la via Nomentana), les deux généraux avaient souscrit aux conditions suivantes de définition de la Città Leonina : «La partie de la ville de Rome qui est délimitée au Sud par les bastions de Santo Spirito, et qui comprend le mont Vatican et le Château Saint-Ange, qui constituent la Città Leonina $»^{20}$. Cette disposition correspond à un souhait de Visconti-Venosta, exprimé dès le mois d'août. Au moment des troubles du 21 septembre, les troupes italiennes s'approchent du ponte Sant' Angelo, mais s'abstiennent de le passer. La position italienne est que le Borgo fait partie de la Città Leonina. C'est le cardinal Antonelli qui demande par écrit l'intervention des troupes italiennes dans le quartier pour rétablir l'ordre ${ }^{21}$. Le 23 septembre Cadorna rappelle par écrit à Antonelli qu'il est prêt à se retirer du Borgo, qu'il considère toujours comme faisant partie de la Città Leonina. Le 25 Antonelli demande à ce que les Italiens restent au Borgo, et occupent le Château Saint-Ange, où des armes et un dépôt de munitions étaient restés sans surveillance ${ }^{22}$. Cadorna s'exécute, mais semble encore considérer que le Borgo n'est pas italien. L'épisode du plébiscite confirme cette hypothèse.

Dans les semaines qui suivent, comme le montrent les mémoires du commissaire Manfroni et les études de Tullio Aebischer, la définition de la Città Leonina par l'Italie est revue à la baisse. La limite est provisoirement fixée, de manière tout à fait pragmatique et sans aucune sanction légale, à la façade de Saint-Pierre. 
Le décret d'annexion du 9 octobre 1870 prévoit à son article trois qu'une loi vienne définir les garanties territoriales accordées au Siège pontifical. La deballatio de l'Etat pontifical s'accompagne donc de l'énoncé de la volonté de régler officiellement la question territoriale. La loi des Garanties de 1871, immédiatement refusée par le Pape Mastai, qui devient loi fondamentale du Royaume en 1878, confirme l'extraterritorialité de la Città Leonina.

Dans ce contexte, les mémoires du commissaire Manfroni fournissent un certain nombre d'informations d'ordre politique et spatial, qui permettent tant de préciser les étapes de la lente prise de possession de la rive droite du Tibre par l'administration italienne, que de souligner le flou persistant qui y a présidé.

Ces mémoires sont ainsi très révélatrices de la gestion prudente et empirique adoptée par le gouvernement italien. Souvent citées pour illustrer la façon dont le Ministère de l'Intérieur apprend peu à peu à décrypter les informations qu'il obtient au sujet des intentions de Pie IX et du Vatican, elles peuvent également être soumises à une lecture d'ordre spatial, dans le but de suivre au travers du récit de Manfroni, commissaire de police dans les quartiers de Trastevere puis du Borgo, la définition progressive entre Italie et Saint-Siège, des aires respectives de compétence administrative et de souveraineté sur l'espace urbain. 
Les mémoires de Giuseppe Manfroni, éditées par son fils, l'historien Camillo Manfroni ${ }^{23}$, en 1920, permettent de confirmer certaines intuitions quant à l'absence d'une position ferme de la part des autorités italiennes au départ, et quant à la mise en place progressive d'une réponse administrative et politique à l'incertitude initiale.

Cette incertitude repose sur une sensation qui marque toute la décennie 1870 dans les rapports entre Etat italien et Vatican, et qui s'impose aux troupes italiennes dès le 20 septembre : toute incursion au-delà du Tibre est perçue comme une provocation face au Vatican. Dès le 20 septembre, la rive droite est perçue comme d'essence éminemment catholique. C'est un caractère qui en marque le destin pour de nombreuses décennies. Dans les années 1880, les projets d'urbanisation du quartier des Prati di Castello sont ainsi tous rejetés au prétexte que construire les nouveaux quartiers de la capitale du royaume sur cette rive serait une provocation face au monde catholique. Au mois de septembre 1870 , ce sont déjà les soldats que l'on hésite à faire traverser le fleuve, et, dans les années qui suivent, la crainte de heurter trop frontalement les susceptibilités et les intérêts du monde catholique et de la Papauté préside à la plupart des décisions relatives à la transformation de l'espace urbain dans cette partie de la ville. Lorsque des projets sont publiés, ils émanent généralement de la gauche garibaldienne, et contiennent de fortes connotations anticléricales. 
Manfroni, fonctionnaire de police arrivant de Gênes, où il était en poste à la Questura, est nommé à la Presidenza du Rione Trastevere, vieille institution urbaine pontificale, héritée de l'Ancien régime et que l'on hésite à supprimer. Sa nomination à ce poste correspond à une volonté ministérielle de ne pas laisser le domaine de la sécurité publique aux mains d'exilés politiques rentrés avec les troupes italiennes. Le commissaire le note dès son arrivée : la question de la cité Léonine est loin encore d'être résolue à la fin du mois de septembre 1870 , et les autorités militaires italiennes hésitent plusieurs jours à faire passer les ponts sur le Tibre à leurs troupes.

C'est bien sûr le Ponte Sant'Angelo, face au Château Saint-Ange, qui pose le plus de problèmes :

«Seppi dunque che la questione della Città Leonina era tutt'altro che risoluta; che fino al giorno 22 i nostri soldati non avevano passato il Tevere a Ponte Sant'Angelo; che nei pressi di San Pietro erano accuduti dei tafferugli tra cittadini e soldati del Papa $»^{24}$

Les soldats ne passèrent le Tibre en direction du Vatican qu'à l'occasion de la répétition de troubles à l'ordre public durant les jours suivants, du fait de manifestations anticléricales près de Saint-Pierre. Mais la mesure est alors considérée comme provisoire : " Era opinione di quelli ufficiali che i nostri avrebbero ripassato il fiume per lasciare a Pio IX un lembo di terra intorno al Vaticano ${ }^{25}$. Manfroni, nommé dans un des deux quartiers de la ville, avec le Borgo, à se trouver sur la rive droite du Tibre, comprend d'emblée 
l'aspect délicat de la situation. Il est placé sous l'autorité directe du général Masi, commandant militaire de Rome et de sa province, en charge de la sécurité publique, et ancien défenseur, notent avec effroi les journaux catholiques, de la République romaine de 1849. Bien vite cependant, il apparaît que c'est au cabinet du Ministre que Manfroni en réfère directement. Face au Vatican, les parcours de la hiérarchie sont abolis : le commissaire est l'interlocuteur direct du cabinet du ministre.

Le rione de Trastevere dont Manfroni à la charge, reprend les limites spatiales du rione de l'administration pontificale. Le premier jour, le commissaire se procure une carte, et tente d'y dessiner les frontières de sa zone de compétence. Il note dès son arrivée que le quartier touche la Città Leonina le long de la via della Lungara, mais aussi qu'il s'étend jusqu'à la mer. C'est là un des points cruciaux du choix qui se présente au gouvernement italien : laisser au Pape les quartiers de la rive gauche revient à lui laisser aussi une façade maritime, gage de cette indépendance que l'Etat italien se fait fort de préserver pour le souverain pontife. Mais laisser à la Papauté des quartiers habités serait aussi lui laisser des habitants, italiens délaissés par le processus d'unification, encombrants sujets d'un Etat pontifical que tout le monde préfèrerait inhabité, ou du moins juridiquement dépourvu de sujets.

Les opérations militaires du 20 septembre 1870 avaient ainsi laissé en suspens de nombreuses questions quant à l'espace urbain. La sensation que 
transmettent les mémoires de Manfroni est que l'administration provisoire du Général Cadorna était résolue à ne pas prendre de décision en la matière et à attendre la mise en place de la Luogotenenza, la lieutenance du Roi, institution destinée à succéder aux instances militaires temporaires. Les troupes italiennes prennent certes possession de la rive droite du Tibre, peutêtre d'ailleurs à la faveur d'une demande de protection, vite retirée, de la part de Pie IX, mais restent discrètes sur cette rive. Une partie des troupes pontificales défaites le 20 septembre s'était réfugiée au Vatican, et la présence des troupes italiennes à proximité avait pour principale fonction d'éviter les incidents entre anticléricaux, susceptibles de manifester au pied des murailles et soldats pontificaux, tout aussi susceptibles de répondre depuis leur place forte.

Les mémoires de Manfroni montrent clairement que, dans les premiers temps, autour du moment du passage de pouvoir, le 9 octobre, entre l'administration militaire de Cadorna et la Luogotenenza de La Marmora, le sentiment était que les soldats italiens allaient se replier en arrière du Tibre, au moins au niveau du Château Saint-Ange, laissant au Pape une portion de territoire plus large, entre le Tibre et la mer. C'est dans ce contexte qu'eut lieu le plébiscite du 2 octobre. Sur ce point, les mémoires de Manfroni permettent de mieux comprendre la complexité du contexte. La question du vote de la rive droite au plébiscite est intimement liée à l'incertitude territoriale. 
Pour Trastevere, il semble que les réticences aient été rapidement emportées par la certitude de ne point trop heurter en ces lieux la susceptibilité vaticane. Mais pour le Borgo, il en alla différemment. Puisque que l'on ne savait pas encore si le Borgo serait italien, ses habitants devaient-ils voter? Manfroni, dans ses mémoires, oppose à l'ordre dans lequel le vote s'est déroulé à Trastevere, sous la présidence du prince Francesco Pallavicini, la confusion du Borgo. Au-delà de l'autosatisfaction du commissaire pour le devoir accompli, dont forcément les mémoires se font l'écho, on peut y voir une indication intéressante. Au Borgo, l'Italie n'a pas imposé son ordre administratif nouveau, et a non seulement gardé la circonscription de police telle qu'elle était sous le gouvernement pontifical, mais a même confirmé dans sa charge le Presidente del rione, homme de confiance, évidemment, du Vatican.

L'administration italienne, qui, pour toute la ville a repris la division territoriale précédente, et dans ce cadre organise le plébiscite de ratification du rattachement de Rome au Royaume, dans le même temps hésite sur l'appartenance d'un de ces rioni, à l'Italie elle-même. Doit-on faire organiser au fonctionnaire pontifical encore en poste à cet endroit le plébiscite de rattachement de la ville, et donc du quartier éventuellement, à un Etat dont il ne peut reconnaître la souveraineté sur la ville elle-même ?

Il faut dire que ce n'est que le 29 septembre que le Château Saint-Ange a été pris par les troupes italiennes, jour même de la publication du règlement 
pour l'organisation du plébiscite. Au moment donc de la publication de ce règlement, l'administration italienne était loin de contrôler, ne serait-ce que militairement, la rive droite dans son entier, $\mathrm{Du}$ point de vue de la symbolique du pouvoir et de la souveraineté, rien n'était réglé.

Manfroni raconte alors comment on a, d'une part des habitants du Borgo, petite clientèle de la cour pontificale, silencieux, et d'autre part des militants démocratiques et libéraux de ce même quartier, sous la conduite du médecin en chef Bianchi, de l'hôpital du Santo Spirito, scandalisés que le pouvoir puisse à ce point douter de l'appartenance de leur quartier à l'Italie.

C'est donc dans la confusion, et sans instructions précises quant à la légalité du scrutin, que l'on vote au Borgo, hors du cadre administratif normal, qui d'ailleurs n'existe pas, au moment même où les tractations entre le cardinal Antonelli et le baron Blanc, pour l'Italie, semblent en bonne voie au sujet de la cité Léonine. Après avoir fait voter les habitants sur la place Pia, et les malades de l'hôpital, Bianchi organise une procession vers l'autre rive derrière une banderole portant l'inscription «Città Leonina: Si ${ }^{26}$. Ce passage en procession d'une rive à l'autre est symbolique à bien des égards, et on retrouve dans ce cortège toutes les incertitudes territoriales dont est chargée la ville. Le Borgo, rione urbain renvoyant à l'ancienne nomenclature des quartiers de la ville, est appelé Città Leonina, expression qui désigne une aire géographique attribuée au Pape par l'acte de capitulation. On passe le fleuve, pour apporter la nouvelle sur la rive 
« italienne ». Le si renvoie au résultat d'un vote qui n'a pas eu de cadre légal. La banderole elle-même résume les ambiguïtés de la situation.

Le 9 octobre La Marmora prend ses fonctions à la Luogotenenza del $\operatorname{Re}^{27}$ Pour cette période, Manfroni note, dans la définition de l'aire de souveraineté laissée au Pape, un moment d'incertitude plus grande encore que dans les deux premières semaines :

« Una strana anomalia impressionava la cittadinanza : nella Città Leonina perdurava l'antica polizia papale, o almeno le funzioni della sicurezza pubblica erano ancora esercitate dall'antico presidente, che era stato confermato in ufficio; di guisa che si ripeteva sempre più insistente la voce che le sorti di quel rione fossero ormai decise, e che Pio IX avrebbe avuto il possesso di una striscia di terre fino al mare $»^{28}$.

On le voit, dans une hypothèse où la cité Léonine inclurait le Borgo, le mois d'octobre était encore loin d'apporter des certitudes territoriales, et même si le nouveau supérieur de Manfroni, le questore Berti, et le conseiller de La Marmora Gerra démentent, le fait que Manfroni rapporte l'hypothèse comme reflétant un sentiment diffus est significatif.

Notons aussi que les forces de sécurité publiques italiennes ont eu bien du mal à imposer leur autorité quotidienne aux habitants. Manfroni le signale pour sa propre circonscription de Trastevere, où il a toutes les peines du monde à substituer son autorité à celle des gendarmes pontificaux. Pour tout le mois d'octobre 1870 , il souligne que ceux-ci continuent de patrouiller en 
civil $^{29}$. Au Borgo la sécurité publique est restée ce qu'elle était sous le gouvernement pontifical : «Seppi anche un'altra notizia che mi riempi di meraviglia : secondo il mio informatore, il governo pontificio continuava a sorvegliare il contegno dei Romani per mezzo di pattuglie di gendarmi, che, vestite in borghese, frequentavano i luoghi pubblici, ascoltando, prendendo nota dei discorsi e degli atti, e riferendo ogni cosa all'ex-ministro della polizia, che continuava a funzionare in segreto, aspettando di poter funzionare in pubblico $»^{30}$

L'affirmation en ville de l'ordre administratif nouveau est donc lente et laborieuse, et les forces de police, dont les circonscriptions ont pourtant repris le schéma territorial ancien, peinent à imposer aux habitants l'évidence de leur autorité. Le Vatican tente discrètement de maintenir aussi longtemps que possible une forme de contrôle sur la ville, le temps de comprendre le sens des événements en cours, et de définir d'autres stratégies de contrôle du territoire urbain de la capitale en devenir d'un Etat honni : insertion politique dans les rouages de la municipalité et gestion orientée de sa confortable emprise foncière essentiellement.

Mais dans les premières semaines de la présence italienne, on en reste à la dimension plus modeste de la patrouille en civil de gendarmes pontificaux.

C'est peut-être un événement qui a lieu sur la rive gauche qui précipite et clarifie le statut de la rive droite et du Borgo : la prise «manu militari », comme le souligne Manfroni, le 8 novembre du Quirinal, palais de résidence 
de Pie IX jusqu'au 20 septembre, envenime en effet les relations entre le gouvernement italien et le Saint-Siège, et met fin aux tractations d'ordre territorial pour le contrôle de la ville. Le Presidente pontifical du rione Borgo est remplacé, mais la confusion quant au titulaire du poste demeure jusqu'en décembre ${ }^{31}$.

Avait été nommé Sernicoli, protégé de Masi. Mais rapidement ce fonctionnaire est débordé par la situation. Le 8 décembre 1870, des cléricaux parviennent manifester place Saint-Pierre, au cri de «viva Pio IX Papa $R e »^{32}$, ce qui évidemment ne peut plaire au questore Berti. Cet incident, au passage, nous indique que c'est à la police italienne qu' incombait l'ordre public sur la place Saint-Pierre, qui fait partie du rione Borgo.

Sernicoli, jugé trop proche des anticléricaux, est remplacé par Manfroni luimême, qui prend en décembre 1870 la direction de la sécurité publique dans le rione Borgo. Charge à lui d'affirmer la stabilité et la viabilité de la présence italienne dans un quartier dont le sort est resté en suspend pendant plus de deux mois. Les instructions du questore Berti à Manfroni sont claires : protéger les cléricaux des assauts « démocratiques », démontrer que le Pape est libre, calmer les libéraux ${ }^{33}$. A partir de ce moment, le gouvernement entend mettre un frein aux agissements de la gauche anticléricale, et ne pas tomber dans le piège d'une détérioration encore plus grave des relations avec la Papauté. Gerra, conseiller du Luogotenente del 
Re La Marmora donne des instructions très précises de fermeté au commissaire. Il lui demande de se considérer comme à «un posto di confine $»^{34}$, sur une frontière.

A cette date, c'est-à-dire décembre 1870, il est précisé que les soldats italiens ne pourront pénétrer au-delà de la Porta di Bronzo. Dans les mémoires de Manfroni, le terme de «confine » revient plusieurs fois, de même que la remarque sur la poursuite des patrouilles des gendarmes pontificaux dans le Borgo. Le bureau du commissaire devient de plus « una piccola agenzia diplomatica » pour les contacts entre gouvernement italien et Saint-Siège. Le commissaire est chargé de manière officieuse de nouer le plus de contacts possibles avec les «amici dell'altra sponda $»^{35}$. Notons dans cette expression que, bien que responsable d'un quartier de la rive droite, Manfroni considère encore implicitement le fleuve comme frontière. $\mathrm{Au}$ cours des dernières semaines de 1870 , c'est la question du voyage du roi, dont Manfroni signale qu'il est ardemment réclamé par les « patriotes », qui occupe les esprits, et relance la question de l'affirmation symbolique de la souveraineté sur la rive droite du Tibre. La question est de savoir si le roi se rendra sur la rive droite du fleuve. Mais pendant de longues semaines, la décision d'organisation du voyage est différée. C'est l'inondation qui frappe la ville à la Noël de 1870 qui contraint le souverain et ses conseillers à mettre fin aux tergiversations. 
La crue du Tibre est effet la pire qu'ait connu la ville depuis de nombreuses décennies, et Manfroni raconte comment les quartiers de la rive droite sont particulièrement touchés par les eaux du fleuve. A cette occasion, il décrit divers petits événements qui continuent de nous informer sur la définition des rapports spatiaux et territoriaux entre Papauté et Italie. Ainsi, un bataillon de soldats italiens reste-t-il prisonnier des eaux dans le Château Saint-Ange, ancien symbole du pouvoir pontifical sur la ville. Il n'y avait pour eux comme seule issue que la galerie couverte qui relie l'édifice au Vatican. Mais face à un refus des autorités pontificales de voir des soldats italiens entrer au Vatican, ils sont contraints de sortir du Château par la grande échelle des pompiers ${ }^{36}$.

C'est le 30 décembre que vient, depuis Florence, Victor Emmanuel, dans une visite aux inondés qui efface les hésitations des semaines précédentes. Mais le souverain se garde bien de passer le Tibre, et reste sur la rive gauche. Manfroni écrit :

«Si sperava che il Re venisse anche in Borgo : ma io pensai subito che su questo punto non potevo farmi illusione e che un certo riguardo alla persona del Pontefice avrebbe consigliato al Re di non passare il ponte. E non mi ingannai : avendo mandato a pregare il questore che mi informasse qualora il re fosse venuto, ne ebbi in risposta che, dovendosi trovare a Firenze per il ricevimento di Capodanno, Sua Maestà non poteva trattenersi 
a Roma se non poche ore, e che per conseguenza i quartieri eccentrici non sarebbero stati visitati ${ }^{37}$.

Manfroni, déçu, comprend cependant que son quartier est "politicamente fin troppo centrale ». Le Borgo est donc italien, mais, comme le souligne le commissaire, plus de la moitié de ses habitants sont «adetti o clienti » du Vatican. Quelques semaines seulement après le rattachement, dans une situation encore floue, une visite officielle aurait été un symbole trop affirmé de souveraineté. Malgré cette réserve royale cependant, il paraît acquis à la fin de l'année 1870 que le Borgo restera italien.

La seule incertitude d'ordre territorial qui demeure en 1871 concerne la place Saint-Pierre et la Basilique, qui se trouvent hors des murailles du Vatican. Il semble, d'après les mémoires de Manfroni, que la police italienne considère la place comme faisant partie de sa circonscription. Elle n'y intervient cependant qu'avec le plus grand tact, et toujours dans le but de circonscrire les provocations anticléricales. Quant à la Basilique, il apparaît que Manfroni ne sait pas bien, et y patrouille lui-même encore en civil, laissant le lieu, bien que partie intégrante du rione Borgo, hors de toute affirmation symbolique d'une éventuelle souveraineté italienne.

A partir de 1871, une des tâches principales de Manfroni est d'ailleurs de recueillir, grâce à ses contacts « di là dal confine », des informations sur ce qui se passe au Vatican pour le compte du gouvernement italien. Il fait tout dès lors pour se renseigner sur la consistance des rumeurs relatives à une 
éventuelle fuite, puis maladie, de Pie IX. Les enjeux sont désormais différents sur le plan politique et diplomatique. Quant au contrôle de l'espace urbain de la nouvelle capitale, il ne se joue plus sur le plan de la police, mais désormais sur celui de la spéculation immobilière, de la vie politique municipale et de la tutelle sur les grands chantiers de modernisation de la ville.

Le souci principal du gouvernement italien est de ne pas provoquer la fuite de Pie IX, et de tenir une ligne de conduite marquée par la volonté d'apaisement. Malgré la présence du bateau français Orénoque à Civitavecchia, jusqu'en 1874, destiné à accueillir Pie IX en cas de fuite, le souverain pontife se résigne à demeurer à l'intérieur des murs du Vatican, et à organiser depuis ce réduit territorial la protestation contre l'usurpation. Il en va autrement avec l'élection au siège pontifical de Joachim Pecci en 1878. Léon XIII décide en effet rapidement d'envisager la possibilité d'une fuite de Rome ${ }^{38}$. Jean-Marc Ticchi a montré qu'à partir de 1880-1881 le Pape, face au péril plus au moins sublimé d'un triomphe de l'élément révolutionnaire, crée une commission chargée d'examiner les voies de fuite. Malte, l'Espagne, le Tyrol et Trieste sont les destinations envisagées. La translation des restes de Pie IX à Saint-Laurent hors les murs en juillet 1881, et les incidents auxquels elle donne lieu, relancent la question. La campagne menée par les garibaldiens en vue de l'abrogation de la loi des Garanties donne de nouveaux arguments au Pape. Après une accalmie en 1884, 
l'hypothèse revient sur le devant de la scène pontificale en 1888-1889, au moment de l'affaire de la statue de Giordano Bruno érigée à Campo de' Fiori. Crispi serait favorable à un départ du Pape. Mais à partir du début des années 1890 il n'en est plus question.

Rien ne change jusqu'en 1914, année où les projets du journaliste et député allemand Erzberger relancent brièvement le débat, autour de l'idée de la concession par l'Italie d'un territoire incluant une façade sur le Tibre. Le projet prévoit la mise en place d'une commission dans laquelle l'Espagne serait médiatrice ${ }^{39}$. Des négociations officieuses sur la question territoriale ont de nouveau lieu en1919, entre Mgr Kelly, Brambilla et le cardinal Gasparri. La chute du gouvernement Orlando les interrompt ${ }^{40}$. Un accord semblait pourtant très proche, avec la reconnaissance du Vatican comme Etat souverain.

Avec Nitti, les discussions continuent, le cardinal Gasparri et Benoît XV souhaitant ardemment arriver à un accord. Rien n'aboutit cependant, dans un contexte politique extrêmement instable du côté italien.

En 1925, Mussolini rouvre la question de la loi des Garanties. Les négociations commencent l'année suivante. On pense un moment insérer Villa Doria Pamphili dans le territoire pontifical. Le 3 octobre 1926, une visite sur les lieux est organisée, tout autour du Vatican. Pie XII souhaite se voir attribuées des zones non peuplées le long de la via Aurelia. Son projet est d'augmenter la superficie de terrain à disposition de la Papauté sans 
perdre le bénéfice de constituer une entité étatique dépourvue d'habitants citoyens. A la fin de l'année 1926, l'ingénieur Cozza, président du Conseil supérieur des Travaux publics, est chargé de la rédaction d'un projet et d'un $\operatorname{plan}^{41}$. En février 1927, Mgr Borgongini remet à Barone, pour la partie italienne, la carte des possessions que voudrait se voir attribuées le SaintSiège. Mais en 1928, le gouvernement fasciste décide de ne rien céder de plus, et de limiter le nouvel Etat au territoire que de facto le Pape contrôle depuis 1870. Le 26 mai, Barone remet à Pacelli, frère de Pie XII, la carte de la zone concédée en souveraineté, et de celle donnée en propriété extraterritoriale. A partir du début de l'année 1929, et de la mort du négociateur Barone, Mussolini prend directement en main le dossier. Sur demande de la Papauté, on ne parle plus que du Vatican pour la zone de souveraineté. Les bâtiments adjacents, comme le Palais du Saint Office, sont en extraterritorialité. La place Saint-Pierre fait partie du territoire du Vatican, mais est ouverte au public sous la juridiction des autorités italiennes $^{42}$. Les accords du Latran sont signés quelques jours plus tard ${ }^{43}$. Une commission technique italo-vaticane est chargée de la définition cartographique de la frontière ${ }^{44}$. Comme le souligne Tullio Aebischer, contrairement à la pratique diplomatique habituelle, dans le Traité du Latran de 1929, la frontière n'est pas décrite textuellement avec précision. La carte mise en annexe au Traité, bien qu'ayant une échelle du $1 / 5000^{\mathrm{e}}$, ne propose pas de visualisation de la limite. La commission mixte mise en place pour 
l'exécution du Traité se trouve donc devant des difficultés d'interprétation. Ce n'est qu'en 1933 que les derniers points litigieux sont réglés.

La question de la limite de l'emprise territoriale pontificale à Rome a ainsi donné lieu à de longues incertitudes. Si un modus vivendi est rapidement trouvé, notamment grâce à l'habileté, pour les premières phases, d'un personnage comme Manfroni, il n'en reste pas moins que la résolution formelle de la question a longtemps été impossible, à cause tant des enjeux adjacents liés aux rapports de l'Etat italien au monde catholique, que de la forte connotation symbolique de chacune des portions de l'espace urbain concernées. La compréhension des enjeux territoriaux à Rome doit par ailleurs inclure une autre dimension, relative à l'emprise foncière de la Papauté dans la capitale de l'Etat italien. Le chapitre de Saint-Pierre est en effet un des plus importants propriétaires fonciers de la ville, et si on y ajoute les propriétés de la noblesse dite noire, qui se déclare fidèle à Pie IX, on comprend que, pour la construction de sa capitale, l'Etat italien ne puisse se contenter d'œuvrer sur le terrain seul de la souveraineté politique. En tant que propriétaire privé de nombreux terrains à construire, le Vatican peut encore, au-delà des années 1870 , jouer un rôle dans les transformations de la ville.

Quant aux mémoires de Manfroni, elles offrent pour toute la fin du pontificat de Pie IX et pour celui de Léon XIII une vision originale de la vie romaine : à la fois ferme et diplomate, le commissaire gère d'année en 
année, campé sur son poste de frontière, les incidents d'un quotidien fait souvent de provocations et de rumeurs, mais aussi de connivences et de précoces tentatives de conciliation.

Denis Bocquet

${ }^{1}$ Voir : G.B. Calogero, Le operazioni militari per l'occupazione dello Stato pontificio e la presa di Roma. Luglio-20 Settembre 1870, dans Studi romani, 1970, p. 340-355.

${ }^{2}$ Idem

${ }^{3}$ Idem

${ }^{4}$ Idem

${ }^{5} \mathrm{~T}$. Aebisher, Le ipotesi territoriali nella questione romana dal 1870 al 1929 , dans Rassegna Storica del Risorgimento, Lugio-Settembre 2000, p. $411-430$.

${ }^{6}$ C. Fiorentino, La questione romana intorno al 1870. Studi e documenti, Rome, Archivio Guido Izzi, 1997, 346 p., p. 13.

${ }^{7}$ Voir : C.M. Fiorentino, Dalle stanze del Vaticano : il Venti Settembre e la protesta della S. Sede. 1870-1871, dans Archivium Historiae Pontificae, 1990, p. 285-333.

${ }^{8}$ Cette circulaire a pour titre " Dalle stanze del Vaticano, 20 Settembre 1870 ». Citée par Fiorentino (C.M.), loc. cit., p. 289. Le titre des mémoires 
du commissaire Manfroni, vraisemblablement donné par son fils, Sulla soglia del Vaticano, dont il sera question plus loin, s'en veut une sorte d'écho depuis le côté italien.

9 Voir: B. Brennan, Visiting 'Peter in Chains': French Pilgrimage to Rome, 1873-1893, dans Journal of Ecclesiastical History, vol 51, 4, 2000, p. 741-765. Ce type de pèlerinage revendicatif marque toute la fin du pontificat de Pie IX, ainsi qu'à partir de 1878 une part importante de celui de Léon XIII. Encore en 1891 des incidents sont notés entre patriotes italiens et catholiques français. Brian Brennan fait état de contremanifestations italiennes au cri de « vive Sedan ».

${ }^{10}$ Cité par Fiorentino, loc. cit., p. 312.

${ }^{11}$ Voir : A.C. Jemolo, Chiesa e Stato in Italia dalla Unificazione ai giorni nostri, Turin, Einaudi, P.B.E., 1977, 331 p.

${ }^{12}$ Voir : G. Martina, La fine del potere temporale nella coscienza religiosa e nella cultura dell'epoca in Italia, dans Archivium Historiae Pontificae, 1971, p. 309-375.

${ }^{13}$ Voir G. Martina, Roma dal 20 settembre 1870 al 11 febbraio 1929, dans L Fioranti et A. Properi (éd.), Storia d'Italia. Annali 16. Roma, la città del Papa. Vita civile e religiosa dal giubileo di Bonifcacio VIII al giubileo di Papa Wojtyla, Turin, Einaudi, 2000, 1253p., p. 938-973.

${ }^{14}$ Voir Martina, loc. cit., p. 325. 
${ }^{15}$ Voir G. De Rosa et F. Malgeri, L'impegno politico dei Cattolici, dans G.

De Rosa, T. Gregory et A. Vauchez, Storia dell'Italia religiosa. III. L'Età contemporanea, Rome-Bari, Laterza, 1995, 619 p., p. 223-256.

${ }^{16}$ Voir : G. Parisella (éd.), La capitale e lo Stato. Governo centrale e poteri locali a Roma. 1870-1990, Rome, Cripes-Kairos, 1992, 135 p.

${ }^{17}$ C.M. Fiorentino, loc. cit., p. 321.

${ }^{18}$ G. Manfroni, Sulla soglia del Vaticano (1870-1901), Bologne, Zanichelli, 1920, 2 vol., 400-310 p

${ }^{19}$ Décret $n^{\circ} 5903$.

${ }^{20}$ Article I de l'acte de capitulation. Cité par T. Aebischer, loc. cit., p. 412.

${ }^{21}$ T. Aebischer, loc. cit., p. 414.

${ }^{22}$ Idem.

23 Spécialiste d'histoire piémontaise, puis d'histoire navale, il enseigne durant les deux premières décennies du XXe siècle à l'Université de Padoue, avant de devenir, sous le fascisme, spécialiste d'histoire coloniale. Il enseigne à partir de 1925 à la faculté des sciences politiques de Rome. Directeur de la Rivista delle Colonie italiane à partir de 1927, il devient aussi sénateur en 1929.

${ }^{24}$ Manfroni, p. 4.

${ }^{25}$ Manfroni, p. 5.

${ }^{26}$ Manfroni, p. 15. 
${ }^{27}$ I.M. Taviani, L'opera della Luogotenenza a Roma (9 ottobre 1870 - 25 gennaio 1871), dans Archivio della Società Romana di Storia Patria, 1970, p. $73-160$

${ }^{28}$ Manfroni, p. 18.

${ }^{29}$ Manfroni, p. 19.

${ }^{30}$ Manfroni, p. 19.

${ }^{31}$ Manfroni, p. 24

32 Taviani, loc. cit., p. 143.

${ }^{33}$ Manfroni, p. 26.

${ }^{34}$ Manfroni, p.31.

${ }^{35}$ Manfroni, p. 36.

${ }^{36}$ Manfroni, p.40-41.

${ }^{37}$ Manfroni p. 45-46.

${ }^{38}$ Voir : J.M Ticchi, Ubi Roma, ibi Papa: les projets de fuite du Pape hors de Rome sous Léon XIII (1878-1895), dans Rassegna Storica del Risorgimento, Luglio-Settembre 2001, p. 355-400.

${ }^{39}$ T. Aebischer, loc. cit., p. 423. Voir aussi : M. Gamberi, Nazionalismo e Santa Sede negli Anni della grande guerra, dans Il Risorgimento, 1997, 12, p. 83-111.

${ }^{40}$ Idem, p.426. Voir aussi : P. Bellu, Verso la Conciliazione (1903-1921), dans Archivio della Società Romana di Storia Patria, 1959, p. 93-173.

${ }^{41}$ Idem, p. 428. 
${ }^{42}$ Idem, p. 430.

${ }^{43}$ Sur les accords du Latran dans le contexte du conflit pluri-décennal entre Etat italien et Vatican, voir: F. Ruffini, Relazioni tra Stato e Chiesa. Lineamenti storici e sistematici, Bologne, Il Mulino, 1974, 316 p., p. 189 et suivantes.

${ }^{44}$ Voir : T. Aebischer, La commissione tecnica italo-vaticana ed i confini del territorio vaticano (1929-1933), Studi romani, 2000, 1, p. 104-117. 